\title{
Acceptance of Product Placement in Italy: Effects of Personality and Product/Consumer Interactions
}

\author{
Gianluigi Guido (Corresponding author) \\ Dept. of Business, Law and Environmental Studies, University of Salento \\ PO box 73100, Via per Monteroni, Ecotekne Campus, Lecce, Italy \\ Tel: 39-832-332-665 E-mail: gianluigi.guido@unisalento.it
}

Alessandro M. Peluso

Dept. of Economics and Business, LUISS University

PO box 00197, Viale Romania 32, Rome, Italy

Tel: 39-333-392-0697_E-mail: apeluso@luiss.it

Piermario Tedeschi

Dept. of Economics and Business, LUISS University

PO box 00197, Viale Romania 32, Rome, Italy

E-mail: patise@tin.it

Chiara Nicole

Dept. of Psychology, "Sapienza” University

PO box 00185, Viale dei Marsi 78, Rome, Italy

E-mail: nickieC@libero.it

Cristina Lauretti

Dept. of Psychology, "Sapienza" University

PO box 00185, Viale dei Marsi 78, Rome, Italy

E-mail: cr.lauretti@tiscali.it

Adele Caciula

Dept. of Economics and Business, LUISS University

PO box 00197, Viale Romania 32, Rome, Italy

E-mail: liberty_ad@hotmail.it

\begin{abstract}
Product placement refers to the planned and paid insertion of a branded product within a film or any other media that is capable of influencing the attitudes and the beliefs of the audience toward that specific product. The present article reports the results of two experimental studies on the attitudes of Italian consumers toward this practice. Findings show that attitudes change as a function of product type; individual differences, such as gender and age; movie watching frequency; as well as consumers' personality profiles. Product placement in movies is quite recent in Italy, since it became a legal practice only in 2004. A comparison with results from previous studies on product placement acceptance, carried out in the United States, Austria, France, China and Australia, is also discussed.
\end{abstract}

Keywords: Attitude, Personality, Product placement 


\section{Product placement}

A wide consensus exists in marketing literature toward affirming the effectiveness of product placement in movies as an instrument capable of affecting viewers' awareness and attitudes (e.g., Balasubramanian et al., 2006; Brennan and Babin, 2004; D'Astous and Chartier, 2000; Law and Braun, 2000; Russell, 2002). Using this technique, marketers can avoid message reiteration to awaken and capture audiences' attention, as the latter is already active, and can take advantage of the involvement of the public exposed to the communication. It is likely that those who have chosen to see a specific movie will tend to follow its development with the maximum attention, including possible product placement (cf. Dalli, 2003; Gistri, 2006). This represents a powerful tool toward product characterization, as it meets the criterion of realism (cf. Hirschman, 1988; Solomon and Englis, 1994), it is virtually immune from elusive practices such as the frenetic change of TV channels (Olney et al., 1991) and from loss of attention during advertising breaks on television (Lawrence, 1989).

Notwithstanding product placement being a particularly common, commercial practice, the interest in this phenomenon has increased only in the last decade (e.g., Karrh, McKee, and Pardun, 2003) and research in the field is still limited. Four main research trends concerning product placement can be identified. The first trend has focused on the content analysis of the product brand insertions within certain movies (e.g., Avery and Ferraro, 2000; Cowley and Barron, 2008; Kinney and Sapolsky, 1994). The second trend has investigated the effects of product placement on consumers' memory, attitudes and purchasing intentions (e.g. Hong, Wang, and De Los Santos, 2008; Russell, 2002) and its economic returns (e.g., Wiles and Danielova, 2009). The third trend has assessed attitudes of movie viewers and sector professionals toward product placement by using phenomenological approaches such as experiments, surveys and interviews (DeLorme and Reid, 1999; Gould, Gupta, and Grabner-Krauter, 2000). The fourth trend approaches the analysis of product placement insertions from a cultural prospective, that is, the relationship that ties the consumer to the objects and to the symbols of consumption (e.g., Stern and Russell, 2004).

\subsection{Audience attitudes toward product placement}

The first study on the general attitudes of audiences toward product placement (i.e. Nebenzahl and Segunda, 1993) showed that most consumers do not openly object to this practice, considering it as an efficient marketing technique. Yet those who do this advanced ethical reasons by caring of an excessive appearance or influence of some products considered as controversial. For this reason, product placement acceptance can be analyzed from two perspectives: general acceptance and acceptance concerning the insertion of specific products. As for product placement acceptance in general, some consumers consider the product placed in a movie as potentially deceitful or even subliminal to the extent that they advise that it should be restricted or prohibited (cf. Miles Homer, 2009; Rothenberg, 1991).

With reference to the acceptance of specific products, it has been proven that the insertion of product information with strong ethical contents, namely products that raise moral issues, is seen less favorably than that of regular (ethically irrelevant) products, such as cars, sunglasses or toothpastes (Gupta and Gould, 1997). This is because ethically charged products tend to generate moral concerns and judgments in consumers with reference to their consumption and excessive visibility, such as cigarettes, alcohol and weapons (Eisend, 2009).

Attention must be paid on particular aspects that can influence consumers' attitudes toward product placement: namely, the type of product included in a movie, the gender of the consumer, the frequency of film viewed (De Gregorio and Sung, 2010; Gupta and Gould, 1997; Gupta et al., 2000; McKechnie and Zhou, 2003); and, for the first time in marketing research, consumers' personality profiles (cf. Caprara et al., 1993). Taking into consideration these determinants, the results obtained in past studies, such as in Gupta and Gould (1997), and Gupta et al. (2000), confirm that people who most frequently watch movies are more likely to appreciate product placement. These studies also show that gender influences the acceptance of ethically charged products, by revealing that, compared to women, men show a more positive attitude toward these products, being products such as alcohol and guns predominantly used by males.

The aim of this study is to show that consumers' personality traits influence their degrees of acceptance of specific products' placement. Psychology researchers (e.g. Caprara, Barbaranelli, and Guido, 2001; Caprara et al., 1993) posit the possibility of reducing the potentially unlimited set of attributes describing human personality to a bounded and more approachable set of attributes that are able to describe individual personality differences. These attributes may be summarized in more general dimensions related to five main traits according to the Big Five model. These traits are Introversion/Extroversion, which is the subjective aversion/predisposition toward social interaction; Neuroticism/Emotional stability, which refers to the subjective ability/inability to respond to external stimuli while keeping emotions and impulses under control; Agreeableness, which refers to the 
orientation toward compassion and caring about others; Conscientiousness, which refers to the preference for goal-oriented activity; Openness to experience, which refers to the degree of tolerance for new ideas and new ways of doing things. These dimensions have been further synthesized by Digman (1997), who found two higher-order factors of personality, i.e., Alpha and Beta. Digman (1997) suggested that personalities dominated by the Alpha factor is distinguished by high levels of Agreeableness, Emotional stability and Conscientiousness, whereas personalities dominated by the Beta factor tend to be characterized by high levels of Extroversion and Openness to experience. These assumptions explain the results that report a larger approval of ethically charged products by individuals ruled by Beta factor.

\subsection{Cross-cultural differences in consumers' behaviors toward product placement}

Understanding cultural differences is often the primary factor in a successful international advertising campaign (Eisend, 2009; Keegan, 1989; McCracken, 1986), insofar as cultural values influence, and are influenced by, different marketing techniques (Belk and Pollay, 1985; Randazzo, 1993). With particular reference to the Western culture, one can say that mass communication influences consumer behavior well beyond the consequences obtained through advertising campaigns: television, cinema, literature continuously repeat symbols, objects, situations and behaviors that tend to reinforce the ideologies and values that are at the basis of our society (Hirschman, 1988). This is true not only for specific objects, but also for a series of more general notions: lifestyles, values, needs and so on. For these reasons, product placement has consequences on cultural aspects. On the one hand, producers and advertisers select meanings and symbols belonging to the dominant culture in order to ensure an effective communication and, on the other hand, consumers' reactions confirm or modify such cultural aspects and they provide elements for the next stage.

Gould and Gupta's (1997) study was replicated in various countries: France and Austria (Gould et al., 2000), as well as China (McKechnie and Zhou, 2003) and Australia (Brennan, Rosenberger, and Hementera, 2004). The two authors observed that certain products are considered more disconcerting than others from an ethical point of view: cigarettes, alcohol and guns are the least accepted products when placed in a movie, as they instill a sense of anxiety amongst the population. The study by Gould, Gupta, and Grabner-Krauter (2000), simultaneously conducted in France and Austria, validated the soundness of Gould and Gupta's approach (1997) to product placement in relation to three variables: individual differences, products and country of origin. The three countries, US, Austria, and France differ in the way domestic consumers react to product placement in terms of acceptance and purchasing intentions. American consumers have a higher propensity to buy products seen in movies; at the same time, in all three countries, some products are considered less acceptable than others (such as ethically charged products). The study by McKechnie and Zhou (2003) suggested that Chinese consumers accept product placement less than American ones, but equally dislike ethically charged products. Furthermore, individual differences appear to have a minimal impact. Contrary to the American context, in which males are clearly more inclined than females to accept the insertion of ethically charged products in a film, the attitudes of Chinese men and women toward the acceptance of this category of products do not differ substantially. The study by Brennan, Rosenberger and Hementera (2004) showed that Australian consumers accept product placement less than American ones find this practice even less acceptable when applied to ethically charged products.

\section{Methodology}

To achieve the research objective, regarding the examination of the influencing factors in product placement acceptance, six hypotheses were developed in two distinct studies. The first study will focus the determinants analyzed in the study by Gould and Gupta (1997), while the second study will examine the role of consumers' personality in determining their acceptance of product placement.

\subsection{Study 1: Product placement acceptance in general and on the basis of specific variables}

Consistent with the study by Eisend (2009), concerns arising from product placement are mostly related to the type of product inserted. The apprehension will then emerge to be higher or lower according to the controversial nature of the products. Moreover, while neutral products acceptance is not influenced by gender differences, perception changes when referring to ethically charged products, as women are more sensitive about such placements (Milner, Fodness, and Morrison, 1991; Nebenzahl and Segunda, 1993; Van Roosmalen and MacDaniel, 1992). For the above reason, it is possible to draw the following hypotheses.

H1: The product placement of ethically charged products is considered less acceptable than ethically neutral ones. 
H2a: There is an interaction between product and gender with respect to the acceptance of the product positioned in a movie.

$\mathrm{H} 2 \mathrm{~b}$ : Given the interaction between product and gender, males accept the product placement of ethically charged products more than females.

Drawing on Gould and Gupta's (1997) study, the frequency of movies watched is an additional variable that can influence consumers' behavior toward product placement. It is assumed that, together with films, all the elements will be appreciated. According to DeLorme and Reid (1999), young consumers do not only appreciate product placement, but they also expect it to suggest realism and a sense of familiarity. The following hypotheses are offered:

H3: Consumers that watch movies more frequently accept more easily product placement than consumers who watch fewer movies.

H4: Consumers who more readily accept product placement in general have a more favorable behavior in accepting products positioned in films.

\subsection{Study 2: Product placement acceptance on the basis of dominant meta-dimensions}

Based on the Big Five model of personality (Goldberg et al., 1992) as a research tool to investigate consumers' personality profiles, the Alpha and Beta meta-dimensions were supposed to affect the degree of acceptance of specific product placement. It can be assumed that individuals dominated by one of the two higher-order factors have a different view of life. In particular, people driven by a personality characterized by the Alpha factor tend to be more reliable and responsible than people with a personality dominated by the Beta factor, and consequently they are more reluctant to accept the insertion of products referring to the fields of health and public security, such as guns, cigarettes and alcohol. Along with the hypothesis made in Study 1 about the interaction between product and gender, and assuming that people with a personality characterized by the Alpha factor are less inclined to accept the inclusion of ethically charged products, it can be suggested that the female gender is more likely to be dominated by such a factor, because it is more sensitive to moral and social matters. This leads to the development of the following hypotheses.

H5: Ethically charged products are perceived as being less acceptable by consumers with a personality governed by the higher-order factor Alpha, as opposed to those with a personality governed by the higher-order factor Beta.

H6: Given an interaction between product and gender, and being men more favorable toward product placement of ethically charged products, the factor Beta appears to be more representative of the male gender, whereas Alpha seems to be more representative of the female gender.

\subsection{Sample}

A total sample of 604 subjects was used for the two studies. The sample was composed of students recruited at three Italian educational institutions: two universities and a professional school of nursing. The sample consisted of $35.4 \%$ males and $64.6 \%$ females; the young people (aged between 16 and 39 ) were $80.3 \%$ whereas adults (40 years and above) were $19.7 \%$.

\subsection{Procedure}

An integrated questionnaire was developed for the two studies, grounding on that employed in the study by Gould and Gupta (1997). Before answering the questionnaire, participants were invited to watch a short clip, made up of a sequence of four pieces of Italian movies in which the product placement technique appears: some popular brands cars (Porsche), lingerie (Lovable), soft drinks (Coca-Cola), and mobile telephone market (Tim) were easily identifiable. To make the audience aware of the content, each piece was preceded by a window that synthesized the title of the film and the product being placed. The questionnaire contained five different sections. The first section began with a precise definition of product placement, followed by two questions regarding the frequency of movies viewed monthly. The second section included a list of 33 statements in a close-end format aimed to assess respondents' attitudes toward product placement. Each statement was followed by a 5-point Likert-type scale, with $1=$ "Strongly disagree" and 5 = "Strongly agree". Respondents were asked to answer about the following themes: (1) product placement in general; (2) product placement as a potentially subliminal and latent advertising form; (3) the contribution of product placement to the film in terms of perceived realism; (4) examples of advertisements usually seen on television; (5) possible restrictions for certain products described as ethically-charged; and (6) the influence that product placement could have on people. Three further questions were added to differentiate our questionnaire from that used by Gupta and Gould (1997). These questions 
regarded: the impact of movie genres and movie stars on consumers' attitudes toward product placement and the influence of product involvement (McKechnie and Zhou, 2003). The third section of the questionnaire explored the respondents' degree of acceptance toward fifteen different products when placed in a movie for commercial purposes. Their degree of acceptance was measured using a three-point scale, with 1 corresponding to "Not acceptable", 2 to "Indifferent", and 3 to "Acceptable". In this section, the following products were added to the list proposed by Gupta and Gould (1997): mobile phones, lingerie and toothpastes. On the other hand, some product categories, i.e., surf equipment and racing cars, were removed, because they are not widely consumed in Italy. For the purpose of Study 2, regarding product placement acceptance on the basis of dominant meta-dimensions, the fourth section included a list of 25 adjectives widely used in psychological research to describe human personality traits. Respondents were invited to indicate on a 7-point scale, with 1 corresponding to "Not at all characteristic of me" and 7 to "Very much characteristic of me", the extent to which each adjective is capable of describing a specific aspect of their own personalities. The fifth section concluded the questionnaire with social-demographic questions.

\section{Results}

To test $\mathrm{H} 1, \mathrm{H} 2 \mathrm{a}, \mathrm{H} 2 \mathrm{~b}$ and $\mathrm{H} 3$ a repeated measure ANOVA was conducted with the degree of acceptance toward the examined products as the dependent variable and "gender", "movie-viewing frequency" and "products" as independent variables. To conduct the analysis on the "movie-viewing frequency" variable, the sample was divided, using a median split of 5, into two groups: "high movie-goers" (5 or more movies) and "low movie-goers" (less than 5). To test hypothesis $\mathrm{H} 4$, the 33 items regarding the attitude toward product placement were factor analyzed, with a principal component technique as extraction method, thereby extracting four latent dimensions. To test hypotheses H5 and H6 several factor analyses with the principal component method and Oblimin rotation were run on personality data collected using the fourth section of the questionnaire, in order to identify the Big Five dimensions. Later, the analysis proceeded on verifying the existence of the two meta-dimensions Alpha and Beta. Finally, a brief international comparison is being expounded between Italy and the nations were similar studies were conducted.

\subsection{Results of Study 1: Product placement acceptability in general and on the basis of specific variables}

There is a significant difference $(t=3.73, p<.05)$ with regards to the acceptance of the various types of products based on gender: male are more favorable than women to the insertion of ethically charged products, whereas there is no significant difference with regards to the number of movies seen on average in a month. A paired-sample $t$-test highlights how a low acceptability degree emerges from the three ethically charged products (cigarettes, alcohol and guns) unlike the higher degree of acceptability for all the other products. Table 1 shows the presence of different degrees of acceptance within the category of ethically charged products: "guns" are the products less accepted if used in a product placement $(M=1.22, S D=.49)$, followed by "alcohol" $(M=1.44, S D$ $=.62)$ and "cigarettes" $(M=1.45, S D=.63)$. Even amongst the neutral products preferences appear: "fatty foods" ( $M=1.76, S D=.64)$ results as being less acceptable, whereas "healthy products" $(M=2.69, S D=.56)$ is the most accepted. These results are confirmed by the frequency percentage of the acceptance of the various products: $3.5 \%, 7.0 \%$ and $7.3 \%$ for guns, alcohol and cigarettes respectively. The acceptance of "fatty foods" is at $11.8 \%$ whereas that of "healthy products" is at $73.9 \%$. These results therefore confirmed H1.

\section{[Insert Table 1 about here]}

The repeated measures ANOVA showed a significant interaction between gender and acceptance of the various products $(F=13.91, p<.05)$. Therefore, H2a found support.

With a series of univariate ANOVA, one can discriminate the influence of gender on the acceptance of the single products. The results obtained for the ethically charged products, highlight a significant effect for "alcohol" $(F=$ $9.25, p<.05)$ and "guns" $(F=35.10, p<.05)$ but not for "cigarettes". Results of the ANOVA conducted to investigate the interrelation between "gender" and "common neutral products" show only a barely significant effect with regards to "healthy products" $(F=3.90 ; p<.05)$ whereas the ANOVA performed between "gender" and the "neutral products" that have been added only to this study did not show significant results. Means reported in Table 2 show a higher acceptance by males for ethically charged products. With regards to means for neutral products common to all studies, "healthy products" (the only product that resulted to be significant), "soft drinks", "fatty foods" and "sweets and snacks" are more accepted by females; "sunglasses", "video cameras", "stereo systems" and "cars" are more accepted by males. The average values on acceptance of the neutral products added to this study show that males accept "mobile phones" and "computers" more readily, and females accept underwear (or "lingerie") and "toothpaste" more readily, thereby confirming H2b.

\section{[Insert Table 2 about here]}


To verify $\mathrm{H} 3$, three univariate ANOVA have been conducted. Amongst the ethically charged products only "alcohol" presents a significant effect $(F=18.49, p<.05)$. Descriptive statistics in Table 3 show that "high movie-goers" $(M=1.48, S D=.63)$ accept more readily product placement of alcoholic products than "low movie-goers" $(M=1.37, S D=.60)$ as hypothesized. Although no statistically significant differences were found for "guns" and "cigarettes", means of the acceptance of both products follow the same trend as observed for "alcohol". An ANOVA conducted on neutral products showed a significant impact in conjunction with "sweets/snacks" $(F=23.33, p<.05)$ and "sunglasses" $(F=18.49, p<.05)$. People who more often watch movies tend to appreciate more product placement of these two neutral products. The univariate ANOVA conducted on the products that were added into our study showed that only "computer" shows a significant effect $(F=16.97, p<.05)$. Means of acceptance of the various products in product placement according to the frequency of viewed films confirm the hypothesis that those who more frequently watch movies accept more readily product placement. $\mathrm{H} 3$ was, therefore, confirmed.

\section{[Insert Table 3 about here]}

To verify H4, after the factor analysis of the main components, an Oblimin rotation was conducted. The outcome resulted in seven extracted factors; altogether the percentage of variance accounted for by the raw data is equal to approximately $57 \%$. The saturations taken into consideration were those with an absolute value equal to or above .60 and for each of the seven factors a reliability analysis was conducted using Cronbach's $\alpha$. Only the first four factors were considered, as the reliability of other three factors falls below .60, and the following semantic interpretation was given: "Behavior toward product placement in general" ( $\alpha=.81)$, "Behavior toward commercials on television" $(\alpha=.82)$, "Limitations on product placement" $(\alpha=.81)$, "Perceived reality" $(\alpha=.63)$. In addition, another factor analysis was conducted imposing a priori that the number of factors to be extracted should equal to four, thus obtaining a $44 \%$ quota of variance which is almost satisfactory.

Subsequently, three new variables were established (following Gupta and Gould, 1997): Index (average acceptance of all the common products), Index 1 (average acceptance of all ethically charged products) and Index2 (average acceptance of all of the neutral products). These three variables were used to run three different Multiple Linear Regressions using the Stepwise method with the four factors that resulted from the previous factor analysis.

Table 4 containing results from the hierarchical regression model shows the analysis conducted on the "Index" variable (a variable that considers all of the products). It can be observed that the complete model becomes significant $(p<.001)$ and a $\mathrm{R}^{2}$ close to .20 . In addition, it can be deducted that the variable "Perceived reality" detains the highest Beta value, also confirmed by the $\mathrm{R}^{2}$ variation values that lead the "Perceived reality" variable to explain the higher variation percentage of $11 \%$. Table 5 pertains to the analysis conducted on "Index 1 " variable (ethically charged products) that was chosen as the dependent variable. The variable with most $\mathrm{R}^{2}$ variation is "Limitations on product placement" with a value equal to $23 \%$, which also achieves the highest Beta value. Table 6 pertains to the analysis conducted on "Index 2" variable (all neutral products) that was chosen as the dependent variable. The "Limitations on product placement" variable is the only one not included in the equation as it does not meet the probability criteria values for $F$ to be inserted or deleted. The variable that explains the most relevant variation is "Perceived reality" with a value equal to $11 \%$.

\section{[Insert Tables 4-6 about here]}

To summarize, a positive behavior toward product placement in general is correlated not only with a positive behavior toward those products that could be positioned within movies ("Perceived reality") but also highlights the difference between ethically charged products and neutral products. In addition, a positive behavior toward product placement in general reduces consumers' desire to impose limitations on product placement of ethically charged products. H4 was, thus, confirmed.

\subsection{Results of Study 2: Product placement acceptability on the basis of dominant meta-dimensions}

The analysis conducted on the 25 items (attributes describing personality) of the fourth section of the questionnaire was run through the factor analysis that brought to the identification of the Big Five dimensions explaining a cumulated variance of $57.1 \%$. The analysis proceeded on verifying the existence of the meta-traits Alpha and Beta. Imposing the existence of two factors, two latent dimensions were identified as explicating the $40.7 \%$ of variance.

Within the Oblimin rotation, the obtained results have led to interpret Factor 1 as the dimension that symbolizes the Beta meta-dimension. In fact, Factor 1 was loaded by adjectives mostly associated with Introversion/Extroversion and Openness to experience. Factor 2, on the other hand, can be interpreted as the 
Alpha meta-dimension, as it was loaded by adjectives associated with Agreeableness, Neuroticism/Emotional stability, and Conscientiousness. A correlation between the these two meta-dimensions and the Big Five was performed, which denoted the Neuroticism/Emotional stability $(r=-.53, p<.001)$, the Conscientiousness $(r=.64$, $p<.001)$ and the Agreeableness $(r=.86, p<.001)$, to be meaningful correlated with Alpha; and the Introversion/Extroversion $(r=.73, p<.001)$ and Openness to experience $(r=-.78, p<.001)$ to be correlated with Beta. Given the factor analysis and correlation matrix, the existence of the two higher-order factors is supported.

The second step considered the goal of correlating the acceptability degree (high or low) of a certain product to the dominant personality trait. The products used in the third section have been divided into two distinct variables: Index 1 (average acceptability of ethically charged products) represents products such as alcohol, guns and cigarettes; Index 2 (average acceptability of neutral products) stands for products such as soft drinks, healthy food, fatty food, sweets/snacks, and computers.

An ANOVA referring to the whole sample population identified a significant difference among mean values ( $p$ $<.001$ ). Ethically charged products are always less appreciated than neutral products (the average acceptability judgments for Index 1 was 1.37; whereas the average acceptability judgments for Index 2 was 2.27). A correlation analysis was conducted on data regarding the two meta-dimensions (Alpha and Beta). In particular, Beta did not correlate with Index $1(r=.047, p=.267)$, while Alpha significantly correlated with Index $1(r=$ $-.134, p=.001)$.

Furthermore, considering the single products, by carrying out another correlation analysis between the higher-order personality dimensions and the different types of products, the correlations between Alpha and cigarettes $(r=-.097, p=.021)$, guns $(r=-.179, p<.001)$, and healthy products $(r=.086, p<.05)$ were significant. Given these correlation results, another ANOVA was run adopting the two personality meta-dimensions (Alpha and Beta) as the dependent variables and Index 1 as the independent variable (Table 7). The obtained results were significant and consistent with H5, which therefore was supported.

[Insert Table 7 about here]

\subsubsection{Higher-order factors on the basis of gender}

Considering the study of Fernandez and Castro (2004), focused on the relation between gender and the personality traits, H6 was demonstrated first by a correlation and then by an ANOVA. The observation of the correlation matrix between the two Alpha and Beta meta-factors and the gender variable, yields significant results for Alpha $(r=.226, p<.001)$; while no significant result appears for Beta $(r=-.033, p=.437)$. A univariate ANOVA was run with Alpha and Beta as dependent variables and gender as the independent variable. No relevant outcome for Beta was found $(p=.437)$, whereas for Alpha a considerable result turned out $(p<.001)$ as shown in Table 8. It is ultimately possible to assert that H6 is verified only for Alpha factor.

[Insert Table 8 about here]

\subsection{An international comparison}

A comparison between the results in Italy and in other countries where similar studies have been conducted ascertains how the Italian population is the one that least appreciates product placement. The average acceptance scores for product placement are in fact lower in Italy than in the other countries examined for the same product categories (see Table 9), the only exception being "healthy food" which results as less accepted both in China and Australia. There is a large gap between Italy and the other countries examined when the research involves the three ethically charged products ("cigarettes", "alcohol" and "weapons") and two products that are considered ethically neutral in this research but which would normally cause debate ("fatty food" and "sweets/snacks"), and whose acceptability is much lower in Italy than in the United States. This might be explained by the different and ancient gastronomic culture of the Italian population.

\section{[Insert Table 9 about here]}

\section{General discussion and conclusions}

The final goal of this research was to explore in-depth the audience's perception of ethics and acceptance of product placement in movies according to: the product category used for the positioning (particularly regarding the products involved with ethics), gender, number of movies seen, personality traits, and the country of residence. This research advances the idea that among the many primary behaviors and values that the group shares, behaviors related to gender are of primary importance. 
Results showed that all the considered ethically charged products placed in a movie seem to be less accepted than neutral products. This reaction depends on the chances that an individual actually has of encountering these products or encountering people who use them in real life: when such a contact increases, the consumer seems to get 'used' to the products and find them less dangerous from an ethical point of view. Among the neutral products, "fatty food" results as the most controversial product, followed by "sweets/snacks", which is a clear insight of the increasing interest of Italian consumers toward physical well-being.

The placement of all the products examined in this research tends to be more accepted by consumers that more often watch movies. Such a difference can be explained by the tolerant and liberal attitude that frequent movie-goers develop since the movie provides the audience with different points of view and realities. A statistically significant difference has been observed for products such as "sweets/snacks", "alcohol", "sunglasses" and "computers"; regarding the last three items, the trend can be explained by the positive hints that these products imply, in Italy they are considered as status symbols: once inserted, products acquire a social valence and customers might look up to such items being used by a positive character in the movie and believe it to be a shortcut to look more like the character. "Sweets/snacks" probably results statistically significant because of the young age of the people interviewed: especially for young Italians (athletes also), afternoon snacks as part of a traditional and healthy diet.

The research highlights product placement acceptability on a gender basis, especially for some specific products. The results show a statistically significant difference for two of the ethically charged products: "alcohol" and "guns" in movies are more accepted by men. "Cigarettes" have lower results in gender differences but follow the trend of "alcohol" and "weapons". Among the neutral items, only one statistically significant difference is identified: "healthy products" is more accepted by women.

A final international comparison of such variables is being highlighted with reference to a constant lower degree of product placement acceptability in Italy than in other countries. This leads to think that the product placement acceptability differs from one country to another depending on cultural differences as well as on each country's legislative regulations.

The present research has emphasized how the prevalence of one of the higher-order factors identified by Digman (1997) confirms a diverse acceptance of specific products placed within movies, and shows a correspondence with either the male or female gender. The descriptions of the two personality profiles are here exposed.

The personality dominated by the Alpha meta-dimension is strongly correlated to products with ethical implications. In fact, it results negatively correlated to Index 1, in that Alpha meta-dimension clearly renders itself more acceptable in society. This higher-order factor identifies a friendly personality, however it is not less cautious because of this. In fact, purchases are guided by a functional and rational need, enough to have them defined as logic shoppers, since close to a utilitarian dimension (cf. Guido, 2006). The motives that push this personality to act in a socially good way are the fear of the consequences of their actions. A personality dominated by the Alpha trait acts less naturally. The negative relation with Emotional stability reveals that these individuals are concerned with pleasing people, behaving in way that does not represent them correctly but that is normally considered correct. They constantly look for a balance, they are insecure, and for this suffer from an internal conflict. The identification of differences between genders confirms that the correlation between Alpha and the female gender is positive. This result is in line with the dominant trait of Alpha, which is Agreeableness, that includes characteristics like altruism, the ability to take care of people, to be cooperative, cordial, empathetic, and faithful, that are mainly carried by women. This also agrees with the study by Toussaint and Webb (2005) that detects a major empathy in women.

Results for the higher-order factor Beta do not show significant findings. However the non-significance of the results is largely relevant for a description. While the distinct profile of Alpha presents a connection with a less enthusiastic vision of life because it feels the weight of social growth with respect to the shared rules, an individual dominated by the Beta factor is mostly concerned with personal growth. The predominant characteristics are the self-esteem, the attainment of well defined personal goals, and the determination to obtain success. The negative correlation with Openness to experience needs to be interpreted as the purpose of improving oneself, and shutting down interactions with others. Such individuals are concerned with realizing their own desires with vitality and passion, and achieving personal expectations with dynamism and confidence. The relationship with products and consumption is hedonistic (Guido, Capestro, and Peluso, 2007), intended as the research of subjective benefit, thus a personality with Beta meta-dimension can be defined a hedonic shopper. For this reason, product correlation is not significant, but it is anyway positively related with Index 1 and Index 2 . Such a personality is not concerned with the ethical implication, but simply with the advantage that can be 
derived from the use of the product. With respect to the gender variable, the negative correlation, even if not significant, induces to maintain that the personalities dominated by Beta meta-dimension tend to be male, as it was shown in the study by Lippa (1995) that recognized to men a greater ambition for social domination and a greater capacity to affirm their points of view.

The results of this research imply the existence of individual and contextual differences that in Italy affect the perception of a relatively new method of marketing communication like product placement. The current study proved that individuals dominated by the Alpha meta-dimension, mainly the female gender, are more sensitive to the communication involving ethically controversial products, that leads them to be less tolerant than individuals with a Beta meta-dimension dominated personality (mainly the male gender) toward the product placement of products such as "cigarettes", "alcohol" and "weapons".

The research outputs suggest that marketing operators should focus on every potential difference among customers (such as country of residence, kind of product, individual differences) when designing a cross-cultural marketing program, as the results might not always meet expectations. Realizing promotional campaigns for a multicultural audience is also a tough task (McDonnell and Drennan, 2010; Redondo, 2006). Adapting the message to the local culture is usually the safest bet and the possibility of different local strategies should always be considered taking in consideration the place where the film is viewed together with a convenient integration between marketing research and the psychology field in order to improve consumer perception of the products. For example it would be improper to address the two types of personalities without distinction, the product placement intended to affect a personality governed by the Beta higher-order factor should aim at astonishing, at creating incongruity in order to obtain a greater attention. The personality governed by the Alpha meta-dimension, instead, being more reliable, needs to be supported for its values, needs a communication planned to emphasize common social values and would definitively reject an aggressive marketing plan.

Finally, this research has attempted to provide a description of consumers' attitudes toward the ethics and acceptability of product placement. Today it is more and more feasible to differentiate the supply and to segment the market based on socio-demographic or behavioral factors and it is necessary to cautiously place ethical charged products in movies, because a wrong positioning could generate concern.

\section{References}

Avery, R. J., \& Ferraro, R. (2000). Verisimilitude or advertising? Brand appearance on prime-time television. Journal of Consumer Affairs, 3, 217-244.

Balasubramanian, S. K., Karrh, J., \& Patwardhan, H. (2006). Audience response to product placements: An integrative framework and future research agenda. Journal of Advertising, 35, 115-141.

Belk, R. W., \& Pollay, R. W. (1985). Materialism and status appeals in Japanese and US print advertising. International Marketing Review, Winter, 38-47.

Brennan, I., \& Babin, L. A. (2004). Brand placement recognition: the influence of presentation mode and brand familiarity. Journal of Promotion Management, 10, 185-203.

Brennan, S., Rosenberger P. J., \& Hementera, V. (2004). Product placements in movies: An Australian consumer perspective on their ethicality and acceptability. Marketing Bulletin, 15, 1-16.

Caprara, G. V., Barbaranelli, C., \& Guido, G. (2001). Brand personality: How to make the metaphor fit? Journal of Economic Psychology, 22, 377-395.

Caprara, G. V., Barbaranelli, C., Borgogni, L., \& Perugini, M. (1993). Big Five Questionnaire to assess the Five Factor Model. Personality and Individual Differences, 15, 281-288.

Cowley, E. \& Barron, C. (2008). When product placement goes wrong: The effects of program liking and placement prominence. Journal of Advertising, 37, 89-98.

Dalli, D. (2003). Il product placement cinematografico: Oltre la pubblicità? International Marketing Trends Congress, 1-16.

D'astous, A., \& Chartier, F. (2000). A study of factors affecting consumer evaluations and memory of product placements in movies. Journal of Current Issues and Research in Advertising, 22, 31-41.

De Gregorio, F., \& Sung, Y. (2010). Understanding attitudes toward and behaviors: In response to product placement. Journal of Advertising, 39, 83-96.

DeLorme, D., \& Reid, L. N. (1999). Moviegoers' experiences and interpretations of brands in films revisited. Journal of Advertising, 28, 71-95. 
Digman, J. M. (1997). Higher-order factors of the Big Five. Journal of Personality and Social Psychology, 73, 1246-1256.

Eisend, M. (2009). A cross-cultural generalizability study of consumers' acceptance of product placements in movies. Journal of Current Issues and Research in Advertising, 31, 15-26.

Fernández, M. L., \& Castro, Y. R. (2004). Sex differences on the five personality factors in Spanish students. Psychological Reports, 95, 101-106.

Gistri, G. (2006). L'Efficacia dell'anticipazione dell'inserimento all'interno di una strategia integrata di product placement cinematografico. Finanza, Marketing \& Produzione, 4, 57-79.

Goldberg, L. R., Hofstede, W. K. B., \& De Raad, B. (1992). Integration of the Big Five and circumplex approaches to trait structure. Journal of Personality and Social Psychology, 63, 146-163.

Guido, G. (2006). Shopping motives, Big Five Factors, and the hedonic/utilitarian shopping value: An integration and factorial study. Innovative Marketing, 2, 57-67.

Guido, G., Capestro, M., \& Peluso A. M. (2007). Experimental analysis of consumer stimulation and motivational states in shopping experiences. International Journal of Market Research, 49, 365-386.

Gupta, P. B., \& Balasubramanian, S. K. (2000). Viewers' evaluations of product placements in movies: Public policy issues and managerial. Journal of Current Issues \& Research in Advertising, 22, 41-53.

Gupta, P. B., \& Gould, S. J. (1997). Consumers' perceptions of the ethics and acceptability of product placements in movies: Product category and individual differences. Journal of Current Issues \& Research in Advertising, 19, $37-51$.

Gupta, P. B., Gould, S. J., \& Grabner-Krauter, S. (2000). Product placements in movies: a cross-cultural analysis of Austrian, French and American consumers' attitudes toward this emerging, international promotional medium. International Journal of Advertising, 29, 41-58.

Hong, S., Wang, Y. J., \& De Los Santos, G. (2008). The effective product placement: Finding appropriate methods and context for higher brand salience. Journal of Promotion Management, 14, 103-120.

Karrh, J. A., McKee, K. B., \& Pardun, C. J. (2003). Practitioners' evolving views on product placement effectiveness. Journal of Advertising Research, 43, 138-149.

Keegan, W. (1989). Global Marketing Management. (4th ed.). Englewood Cliffs: Prentice Hall.

Law, S., \& Braun, K. A. (2000). I'll have what she's having: gauging the impact of product placement on viewers. Psychology and Marketing, 17, 1059-1075.

Lippa, R. (1995). Gender-related individual differences and psychological adjustment in terms of the Big Five and circumplex models. Journal of Personality \& Social Psychology, 69, 1184-1202.

McCracken, G. (1986). Culture and consumption: A theoretical account of the structure and movement of the cultural meanings of consumer goods. Journal of Consumer Research, 13, 71-84.

McDonnell, J., \& Drennan, J. (2010). Virtual product placement as a new approach to measure effectiveness of placements. Journal of Promotion Management, 16, 25-38.

McKechnie, S. A \& Zhou, J. (2003). Product placement in movies: A comparison of Chinese and American consumers' attitudes. International Journal of Advertising, 22, 349-374.

Miles Homer, P. (2009). Product placements: The impact of placement type and repetition on attitude. Journal of Advertising, 38, 21-31.

Milner, L. M., Fodness, D., \& Morrison, J. (1991). Women's image of guns: An exploratory study. In J.A. Costa (Ed.), Gender and Consumer Behaviour, Salt Lake City: University of Utah Printing Press, pp. 199-208.

Nebenzahl, I. D., \& Segunda, E. (1993). Consumers' attitudes toward product placement in movies. International Journal of Advertising, 12, 1-11.

Randazzo, S. (1993). Advertising as myth-maker: Brand as Gods and heroes. Advertising Age, 64, 32.

Redondo, I. (2006). Product-placement planning: How is the industry placing brands in relation to moviegoer consumption?" Journal of International Consumer Marketing, 18, 33-60.

Russell, C. A. (2002). Investigating the effectiveness of product placement in television shows: The role of modality and plot connection congruence on brand memory and attitude. Journal of Consumer Research, 29, 306-318.

Stern, B. B., \& Russell, C. A. (2004). Consumer responses to product placement in television sitcoms: Genre, sex, and consumption. Consumption, Markets and Culture, 7, 371-394. 
Toussaint, L., \& Webb, J. R. (2005). Gender differences in the relationship between empathy and forgiveness. Journal of Social Psychology, 145, 673-685.

Van Roosmalen, E. H., \& McDaniel, S. A. (1992). Adolescent smoking intentions: Gender differences in peer context. Adolescent, 27, 87-105.

Wiles, M., \& Danielova, A. (2009). The worth of product placement in successful films: An event study analysis. Journal of Marketing, 73 (4), pp. 44-63.

Table 1. Means of products' acceptance levels

\begin{tabular}{|l|c|c|}
\hline Products & Mean & Standard Deviation \\
\hline Healthy Products & 2.69 & .56 \\
\hline Soft Drinks & 2.43 & .71 \\
\hline Sunglasses & 2.31 & .68 \\
\hline Computer & 2.31 & .68 \\
\hline Cars & 2.30 & .69 \\
\hline Toothpaste & 2.28 & .66 \\
\hline Underwear & 2.27 & .67 \\
\hline Stereo system & 2.26 & .66 \\
\hline Video camera & 2.23 & .67 \\
\hline Mobile Phones & 2.13 & .71 \\
\hline Sweets/Snacks & 2.07 & .67 \\
\hline Fatty Foods & 1.76 & .64 \\
\hline Cigarettes & 1.45 & .63 \\
\hline Alcohol & 1.44 & .62 \\
\hline Guns & 1.22 & .49 \\
\hline
\end{tabular}

$\mathrm{N}=604$.

Table 2. Means of the acceptance of the products based on gender

\begin{tabular}{|l|c|c|c|c|}
\hline Products & Males & Standard & Females & Standard \\
\hline Healthy Products & 2.63 & .61 & 2.72 & .52 \\
\hline Soft Drinks & 2.38 & .71 & 2.46 & .71 \\
\hline Sunglasses & 2.31 & .69 & 2.30 & .67 \\
\hline Computer & 2.31 & .69 & 2.31 & .68 \\
\hline Cars & 2.36 & .68 & 2.26 & .70 \\
\hline Toothpaste & 2.22 & .65 & 2.32 & .66 \\
\hline Underwear & 2.26 & .68 & 2.27 & .67 \\
\hline Stereo system & 2.26 & .67 & 2.25 & .65 \\
\hline Video camera & 2.24 & .68 & 2.23 & .66 \\
\hline Mobile Phones & 2.16 & .70 & 2.11 & .72 \\
\hline Sweets/Snacks & 2.03 & .69 & 2.09 & .66 \\
\hline Fatty Foods & 1.73 & .66 & 1.78 & .64 \\
\hline Cigarettes & 1.49 & .63 & 1.43 & .63 \\
\hline Alcohol & 1.54 & .65 & 1.38 & .60 \\
\hline Guns & 1.37 & .63 & 1.13 & .37 \\
\hline
\end{tabular}

$\mathrm{N}=604$. 
Table 3. Means of products' acceptance levels based on the frequency of films viewed

\begin{tabular}{|l|c|c|c|c|}
\hline Products & High movie-goers & $\begin{array}{c}\text { Standard } \\
\text { Deviation }\end{array}$ & Low movie-goers & $\begin{array}{c}\text { Standard } \\
\text { Deviation }\end{array}$ \\
\hline Healthy Products & 2.67 & .56 & 2.72 & .56 \\
\hline Soft Drinks & 2.46 & .69 & 2.40 & .74 \\
\hline Sunglasses & 2.35 & .64 & 2.23 & .73 \\
\hline Computer & 2.35 & .65 & 2.24 & .72 \\
\hline Cars & 2.33 & .65 & 2.23 & .73 \\
\hline Toothpaste & 2.30 & .64 & 2.25 & .69 \\
\hline Underwear & 2.29 & .64 & 2.23 & .70 \\
\hline Stereo system & 2.28 & .64 & 2.21 & .73 \\
\hline Video camera & 2.26 & .65 & 2.18 & .67 \\
\hline Mobile Phones & 2.16 & .70 & 1.99 & .65 \\
\hline Sweets/Snacks & 2.11 & .67 & 1.72 & .58 \\
\hline Fatty Foods & 1.79 & .64 & 1.39 & .60 \\
\hline Cigarettes & 1.48 & .65 & 1.21 & .49 \\
\hline Alcohol & 1.48 & .63 & & .79 \\
\hline Guns & 1.22 & & & .79 \\
\hline
\end{tabular}

$\mathrm{N}=604$.

Table 4. Results of the hierarchical regression analysis

\begin{tabular}{|c|c|c|c|c|}
\hline Step. & Variable & $\beta$ & $t$-value & $p$-value \\
\hline 1. & $\begin{array}{l}\text { Behavior toward commercials on television } \\
\text { Limitations on Product Placement } \\
\text { Perceived Reality }\end{array}$ & $\begin{array}{l}-.236 \\
.251 \\
.366\end{array}$ & $\begin{array}{r}-5.970 \\
6.434 \\
9.656\end{array}$ & $\begin{array}{l}.000 \\
.000 \\
.000\end{array}$ \\
\hline \multicolumn{5}{|c|}{$\mathrm{R}=.306, \mathrm{R}^{2}=.093$, Adj. $\mathrm{R}^{2}=.092, \Delta \mathrm{R}^{2}=.093, \mathrm{~F}=58.789, \mathrm{p}<.001$} \\
\hline 2. & $\begin{array}{l}\text { Limitations on Product Placement } \\
\text { Perceived Reality }\end{array}$ & $\begin{array}{l}.217 \\
.345\end{array}$ & $\begin{array}{l}5.600 \\
9.263\end{array}$ & $\begin{array}{l}.000 \\
.000\end{array}$ \\
\hline \multicolumn{5}{|c|}{$\mathrm{R}=.383, \quad \mathrm{R}^{2}=.147$, Adj. $\mathrm{R}^{2}=.144, \Delta \mathrm{R}^{2}=.053, \mathrm{~F}=35.647, \mathrm{p}<.001$} \\
\hline 3. & Perceived Reality & .340 & 9.392 & .000 \\
\hline
\end{tabular}

$\mathrm{N}=604$. Dependent Variable $=$ INDEX

Table 5. Results of the hierarchical regression analysis

\begin{tabular}{|c|c|c|c|c|}
\hline Step. & Variable & $\beta$ & $t$-value & $p$-value \\
\hline 1. & $\begin{array}{l}\text { Behavior toward commercials on television } \\
\text { Limitations on Product Placement } \\
\text { Perceived Reality }\end{array}$ & $\begin{array}{l}-.194 \\
.531 \\
.196\end{array}$ & $\begin{array}{l}-4.669 \\
14.180 \\
4.703\end{array}$ & $\begin{array}{l}.000 \\
.000 \\
.000\end{array}$ \\
\hline \multicolumn{5}{|c|}{$\mathrm{R}=.109, \mathrm{R}^{2}=.012, \operatorname{Adj} . \mathrm{R}^{2}=.010, \Delta \mathrm{R}^{2}=.012, \mathrm{~F}=6.949, \mathrm{p}<.001$} \\
\hline 2. & $\begin{array}{l}\text { Limitations on Product Placement } \\
\text { Perceived Reality }\end{array}$ & $\begin{array}{l}.495 \\
.177\end{array}$ & $\begin{array}{c}13.536 \\
4.297\end{array}$ & $\begin{array}{l}.000 \\
.000\end{array}$ \\
\hline \multicolumn{5}{|c|}{$\mathrm{R}=.219, \mathrm{R}^{2}=.048$, Adj. $\mathrm{R}^{2}=.045, \Delta \mathrm{R}^{2}=.036, \mathrm{~F}=21.796, \mathrm{p}<.001$} \\
\hline 3. & Perceived Reality & .166 & 4.630 & .000 \\
\hline
\end{tabular}

$\mathrm{N}=604$. Dependent Variable $=$ Index 1 
Table 6. Results of the hierarchical regression analysis

\begin{tabular}{|c|c|c|c|c|}
\hline Step. & Variable & $\beta$ & $t$-value & $p$-value \\
\hline 1. & $\begin{array}{l}\text { Behavior toward commercials on television } \\
\text { Limitations on Product Placement } \\
\text { Perceived Reality }\end{array}$ & $\begin{array}{l}-.200 \\
.079 \\
.353\end{array}$ & $\begin{array}{l}-5.044 \\
1.966 \\
9.311\end{array}$ & $\begin{array}{l}.000 \\
.000 \\
.000\end{array}$ \\
\hline \multicolumn{5}{|c|}{$\mathrm{R}=.318, \mathrm{R}^{2}=.101$, Adj. $\mathrm{R}^{2}=.099, \Delta \mathrm{R}^{2}=.101, \mathrm{~F}=64.270, \mathrm{p}<.001$} \\
\hline 2. & $\begin{array}{l}\text { Limitations on Product Placement } \\
\text { Perceived Reality }\end{array}$ & $\begin{array}{l}.047 \\
.336\end{array}$ & $\begin{array}{l}1.164 \\
8.950\end{array}$ & $\begin{array}{l}.000 \\
.000\end{array}$ \\
\hline \multicolumn{5}{|c|}{$\mathrm{R}=.373, \mathrm{R}^{2}=.139$, Adj. $\mathrm{R}^{2}=.136, \Delta \mathrm{R}^{2}=.038, \mathrm{~F}=25.446, \mathrm{p}<.001$} \\
\hline 3. & Perceived Reality & .039 & 1.042 & .000 \\
\hline
\end{tabular}

$\mathrm{N}=604$. Dependent Variable $=$ Index 2

Table 7. Univariate ANOVA between the Big Two and Index 1

\begin{tabular}{|l|c|c|c|c|c|}
\hline & Sum of Squares & Df & Mean of Squares & F & $p$ \\
\hline Alpha: Between groups & 11.944 & 6 & 1.991 & 2.022 & .041 \\
\hline Beta: Between groups & 3.725 & 6 & .621 & .618 & .716 \\
\hline
\end{tabular}
$\mathrm{N}=604$.

Table 8. Univariate ANOVA between the Meta-dimensions and the gender variable

\begin{tabular}{|l|c|c|c|c|c|}
\hline & Sum of Squares & Df & Mean of Squares & F & $p$ \\
\hline Alpha: Between groups & 28.962 & 1 & 28.962 & 30.467 & .000 \\
\hline Beta: Between groups & .604 & 1 & .604 & .604 & .437 \\
\hline
\end{tabular}

$\mathrm{N}=604$.

Table 9. Average acceptance scores of product placement in various countries for different product categories

\begin{tabular}{|l|c|c|c|c|c|c|}
\hline Product Category & Italy & USA & France & Austria & China & Australia \\
\hline Soft Drinks & 2.43 & 2.93 & 2.84 & 2.87 & 2.82 & 2.49 \\
\hline Cigarettes & 1.45 & 2.04 & 2.14 & 1.95 & 1.79 & 1.56 \\
\hline Alcohol & 1.44 & 2.36 & 2.22 & 2.20 & 2.25 & 1.87 \\
\hline Fatty Foods & 1.77 & 2.83 & 2.78 & 2.28 & 1.84 & 2.15 \\
\hline Healthy Products & 2.70 & 2.91 & 2.74 & 2.91 & 2.62 & 2.60 \\
\hline Guns & 1.22 & 1.94 & 1.66 & 1.58 & 1.52 & 1.75 \\
\hline Sweets/Snacks & 2.07 & 2.87 & 2.74 & 2.73 & 2.31 & 2.39 \\
\hline Sunglasses & 2.31 & 2.86 & 2.87 & 2.89 & 2.54 & 2.51 \\
\hline Video camera & 2.23 & 2.86 & 2.86 & 2.82 & 2.71 & 2.46 \\
\hline Stereos & 2.26 & 2.86 & 1.99 & 2.83 & 2.71 & 2.45 \\
\hline Cars & 2.30 & 2.90 & 2.87 & 2.90 & N.P. & 2.54 \\
\hline
\end{tabular}

$\mathrm{N}$ Italy $=604 ; \mathrm{N} \mathrm{USA}=1012 ; \mathrm{N}$ France $=204 ; \mathrm{N}$ Austria $=240 ; \mathrm{N}$ China $=108 ; \mathrm{N}$ Australia $=146$. 